\title{
Coordinated robotic system for civil structural health monitoring
}

\author{
Uvais Qidwai ${ }^{1}$ * and Muhammad Ali Akbar ${ }^{1}$ \\ ${ }^{1}$ College of Engineering, Qatar University, Doha, Qatar
}

\begin{abstract}
With the recent advances in sensors, robotics, unmanned aerial vehicles, communication, and information technologies, it is now feasible to move towards the vision of ubiquitous cities, where virtually everything throughout the city is linked to an information system through technologies such as wireless networking and radio-frequency identification (RFID) tags, to provide systematic and more efficient management of urban systems, including civil and mechanical infrastructure monitoring, to achieve the goal of resilient and sustainable societies. In this proposed system, unmanned aerial vehicle (UAVs) is used to ascertain the coarse defect signature using panoramic imaging. This involves image stitching and registration so that a complete view of the surface is seen with reference to a common reference or origin point. Thereafter, crack verification and localization has been done using the magnetic flux leakage (MFL) approach which has been performed with the help of a coordinated robotic system. In which the first robot is placed at the top of the structure whereas the second robot is equipped with the designed MFL sensory system. With the initial findings, the proposed system identifies and localize the crack in the given structure.
\end{abstract}

\section{Introduction}

The advancement in modern civil architecture along with the considerable number of natural disasters in the last decades creates an alarming situation for the reliability of the infrastructures. If only weather related natural disasters are considered then total 6,457 events has been recorded from 1995 to 2015, according to the United Nations International Strategy for Disaster Reduction (UNISDR) [1]. It has also been claimed in [1] that every year 205 million people on average were affected by such natural disasters. These disasters may not only caused large number of causalities but also damages the stability of the infrastructure which creates alarming situation for those who survives. Therefore, a regular and highly precise monitoring system is required to provide the quality health assurance of the civil structures [2]. In current tradition a team of experts is required to physically examine the buildings and then prepare the report accordingly. However, this approach is not very feasible because of the recent structural complexities along with their heights which are much more than the old traditional buildings. The recent advances in sensors,

*Corresponding author: uqidwai@qu.edu.qa 
robotics, unmanned aerial vehicles, communication, and information technologies, can be adopted to fulfil the dream of ubiquitous cities where virtually everything throughout the city is linked to an information system by means of wireless networking and radiofrequency identification (RFID) tags. Thus, a more efficient and systematic management of urban systems monitoring including civil and mechanical infrastructure is become possible to achieve the goal of resilient and sustainable societies.

In the past, many different approaches have been adapted to provide frequent/long-term monitoring of the structures. Most common of these approaches is the vibrational analyses, in which an accelerometer will be connected to different joints of the structure and the vibrations recorded by the instrument will be used to determine the damage in the structure [3-6]. However, vibration-monitoring approach cannot be used to assess the slow degradation of the structure [7]. Moreover, increasing the uncertainties caused by different factors like the environmental condition will also decrease the reliability of damage detection using vibration approach [8].

A magnetic flux leakage (MFL) based structural health monitoring (SHM) approach is one of the predominant approach for metallic structures due to their excellent reproducibility and reliability [15]. The magnetic sensor reads constant values of magnetic flux produced by permanent magnet but when it comes in contact with a crack the leakage in magnetic field will be used to identify the crack. This approach has widely been used for cable inspection [15], steel pipes [16] etc.

Digital imaging is considered to be among the promising approach for automated vision based inspection (VBI) which can help to remotely perform the crack analysis. It is currently being the predominant approach to detect the crack on structure [9-10]. The performance of digital imaging is broadly dependent on the way of capturing images as well as the algorithm used for image processing. In [11] Zhu et al. used a personal camera to take the sight images of the bridge. These images will then be processed using edge detection followed by Hough transform to provide the crack identification. The research work has been conducted using the assumption that the concrete columns of each building have a pair of vertical lines with uniform texture and colour. However, the assumption is not applicable for the real world structure.

The similar approach using marker instead of vertical lines has been used for crack identification using unmanned aerial vehicle (UAV) by Ellenberg et al. in [12]. The need of markers can be overcame by using invariant features based image stitching approach. In this approach the set of images obtained before and after the crack will be stitched separately by using the scale invariant feature like SURF, SIFT etc. Thereafter, the final stitched image of the structure before and after crack will be compared to perform the SHM. The approach has been investigated for bridge inspection [11] and tunnel inspection [13]. The image stitching based SHM seems to be promising for the identification of physical and mechanical defects on the surface of the structure. However, the key aspect of such approach lies in the selection of feature detection algorithm which best suits for image stitching and crack identification.

Although the VBI is seems to be the most effective approach for crack identification. However, it can only provide limited information for maintenance planning [14]. The MFL sensor on the other hand can be used to localize the crack but the response time is slower due to the settling time required to from stable magnetic field during movement [15]. Therefore in this research an integration of VI and MFL has been proposed to detect, verify and localize the crack or structural deformation. The VBI is used to provide early identification of the crack presence whereas the MFL will help to quantitatively analyze and localize the crack. The whole system is automated to get more accurate and robust SHM. 
The task of VBI has been achieved with the help of UAV followed by an automated image stitching and anomaly detection algorithm. Whereas the MFL inspection has been achieved with a modular robotic design. In which the first robot is placed at the top of the structure whereas the second robot is equipped with the designed MFL sensory system. The proposed approach can easily be adopted to any metallic structure.

The remaining sections of this paper are organized as follows. Section 2 covers the experimental setup. The proposed approach is described in Section 3. Section 4 is concerned with the simulation results and their discussion. Section 5 concludes the paper.

\section{Experimental setup}

Since the goal of this research work is to develop a coordinated SHM which uses the advantages of visual inspection for early detection along with the Magnetometer based verification approach. Therefore the experimental setup has also been setup to adopt these two approaches and are described in this section.

\subsection{UAV specification}

Visual inspection is considered to be the predominant approach for crack detection and identification [10]. This approach is mainly performing the similar operation as that of a sight inspector and that is the reason it has also been used to assist him in SHM. The images for the vision based approach can either be achieved by a fixed camera as in [11] or by means of a flying UAV as adopted in [12].

In our research the images has been obtained using a commercially available UAV (Phantom Professional-3), as shown in Fig. 1(a). The reason of selecting this UAV is because of its high stability and control mechanism which is ensured through different control and sensing modules such as gyroscope, 3-axes accelerometer and an ultra-sonic sensor [17]. The UAV during inspection process is shown in Fig 1(b).

Phantom drone, with its reinforced structure offered a highly safe and robust design in spite of having a net weight of only $1280 \mathrm{~g}$. The powerful air braking mechanisms of phantom enables it to be stop instantly such that it can hover in one place as soon as the control sticks are released. The most important feature of Phantom Pro. III lies in its 12 megapixels " $4 \mathrm{~K}$ " camera, which can be used to take high-quality images and videos, with a 3-axis gimbal control based advance image stabilization technology which insure highly stable and clear images even during the flight [17].

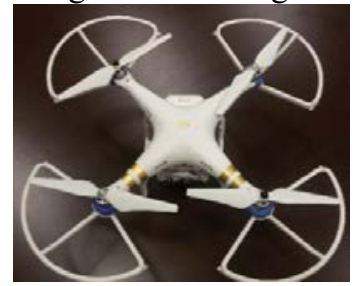

(a)

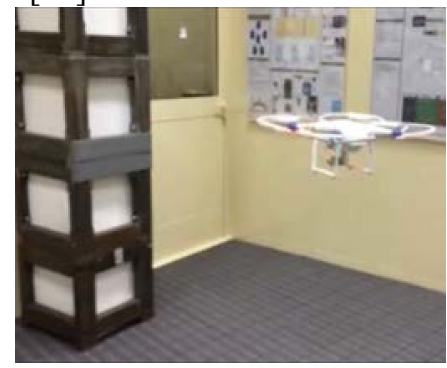

(b)

Fig. 1. Phantom drone in (a) stationary and (b) during inspection. 


\subsection{Hall effect based verification}

The MFL is considered to be the most economical approach for inspecting the metallic structures [16]. Traditionally a hand-held device is used for performing the MFL inspection. These devices are normally huge in sizes and can mostly be used for inspecting the pipelines or the sheets. However, it is very difficult to inspect a building using these devices because of the size and weight. Therefore, in this research an autonomous MFL inspection robot has been presented which is small, flexible and remotely accessible.

The robotic design for this purpose used the concept of abseiling, in which the cliffclimber tighten the rope at the top and then move up and down with the support of the rope. Similarly, the designed robot have two modules connected with each other. The first module of robot (FMR) is placed at the top of the structure and is responsible to assist the other module in inspection by performing all computation tasks related to positioning control, data acquisition etc. The second module of the robot (SMR) is having its own control circuitry, permanent magnets and an array of MFL sensor. The permanent magnets were not only used to provide the magnetic field but are also used to keep a uniform contact between the MFL sensor and the structure specially when the second module moving vertically.

The FMR should be strong enough to hold the SMR and other environmental effects like strong wind, gravitation pull etc. Moreover, it should have a precise motion along with other features like obstacle detection to avoid any collision. However, in this research, a Lab grad scaled version of FMR has been used which is designed using a VEX robotic kit [18], as shown in Fig. 2(a).

The FMR is also equipped with ultra-sonic sensors which can help the FMR to change its path in case of obstacle. The control mechanism of FMR is done with the help of Arduino Mega2560 Microcontroller. It is also responsible to coordinate between the FMR and SMR. A compass and GPS module is also placed in FMR which helps to estimate the target location with respect to the current location.

The SMR is designed in such a way that the size of the robot is small enough to move on all parts of the target structure. It has its own motors and controller as well but the motors are small enough to be easily driven by a $9 \mathrm{~V}$ battery. The permanent magnet along with the MFL sensor are placed at the lower base of the robot, as shown in Fig. 2(b). The $\mathrm{SMR}$ is connect with a wire controlled by the wrench motor placed on the FMR.

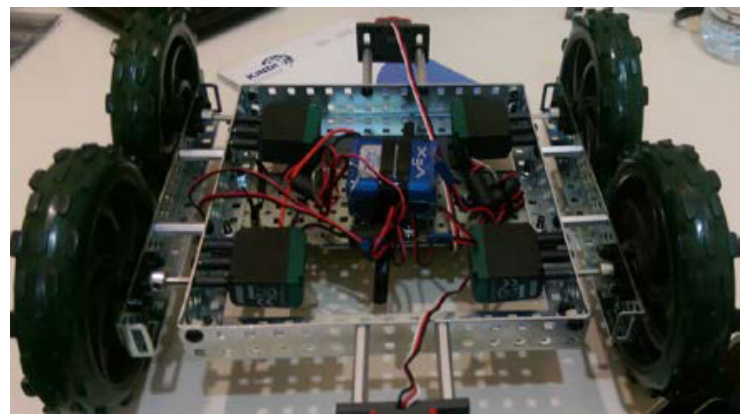

(a)

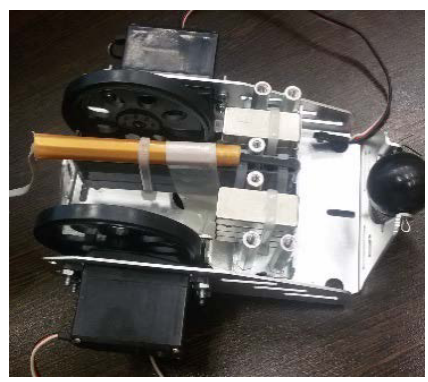

(b)

Fig. 2. The coordinated robot for MFL (a) FMR and (b) SMR.

\section{Proposed approach}

The proposed approach used the advantages of the visual and MFL inspection approach to improve the efficiency of the SHM. Therefore, the usage of both approaches should be 
done in a way that the whole inspection is carried out in an optimal time period. Thus, due to the fast processing of visual inspection, it is done first followed by an MFL based verification approach. The overall inspection process is described in Fig.

\subsection{Visual inspection process}

The visual inspection has been carried out such that the drone will take-off from a fixed point and take images at different heights without changing the GPS coordinate values of start point during flight. After completing the first scan, the coordinates of the GPS will be shifted and same procedure of taking images at different heights will be conducted. The process remain continue until the drone reaches to the starting GPS coordinates. The images which were taken at different heights for particular coordinates are considered as a single set.

Thereafter the image stitching (IS) is applied on individual sets. The process of IS involves a series of steps which were applied on the consecutive images of a particular set, such that one of the image is taken as a reference image (RI) whereas the other one is termed as the current image (CI). The resultant stitched image will be RI for the next consecutive image and then the whole stitching process is applied. The process remain continue for each set until a final stitched image has been obtained from them. The stitched result will be saved in the database with its corresponding GPS values.

The initial step in the IS algorithm is to determine the point of interest (POI) in both RI and CI. There are different algorithm to perform the detection of POI, among them some are already present as MATLAB built-in library function. In this paper we adopted Speeded Up Robust Features (SURF) the most commonly used approaches for POI. It is a scale and rotation invariant point of interest detector which was introduced by Herbert et al. in 2006 [14]. Thereafter, the feature has been extracted around each POI. The whole process of POI and feature extraction is done separately on both images which needs to be registered.

After determining the POI and their corresponding feature vector. The common features between the images have been computed which will help to determine the changes between the CI with respect to the RI. Since the matched features are in actual corresponding to some SURF points in both images. Therefore, after feature matching the corresponding point of SURF which are common in both images is determined with the help of matched features. These points are termed as matched points (MP). It should be noted that the MPs may have some outliers as well, in order to reduce them the RANdom SAmple Consensus (RANSAC) algorithm is performed to exclude the outlier and compute the homogeneousness between two images. Finally, the affine transformation has been applied on CI using the reduced MP so as to align CI with respect to RI. The transformed CI is fused in to RI in a way that only the portion of CI which is not present in RI will be added in to it.

The same procedure of taking and stitching the images of the same structure will be repeated again after few months, depending upon the structural sensitivity as well as the severity of the weather condition around it. The current results will be compared with the stitched images present in the data base and if some anomaly is detected then the HP coordinates (i.e. the GPS coordinates) along with the estimated height for that particular location will be sent to the FMR to proceed the crack verification using MFL.

\subsection{MFL inspection process}

The GPS module present in the FMR will guide the robot about its own location. As soon as Arduino Mega2560 Microcontroller receives the GPS coordinates from the system. It will translate them and compare them with its current location. The need of translation is 
because the FMR is present at the top of the building whereas the drone is flying at particular distance from the building. In order to obtain a correct translation the drone should remain at particular distance form in structure during the whole scanning process. The robot will take its direction based on the comparison result between its current GPS coordinates and the translated received GPS coordinates. As the robot moves it will keep checking the current GPS values and take decision accordingly.

Since there might be some temporary or permanent obstacle present on the roof for decoration purpose. Therefore an ultrasonic range sensor has been used such that when the robot come close to an obstacle at defined distance the sensor will guide the robot to change its path and as soon as the obstacle is disappeared from the sensor range the robot will again start checking the GPS value to reach to its target destination.

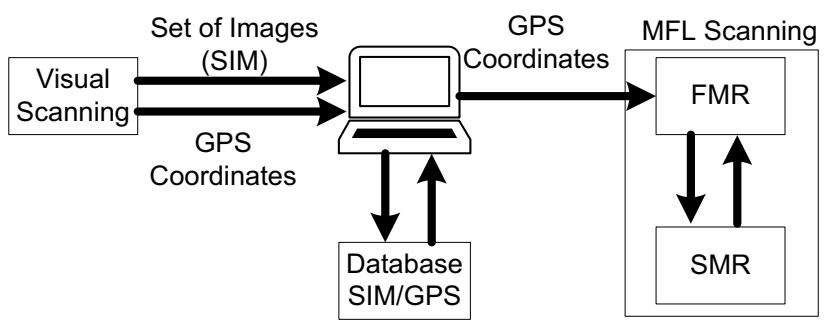

Fig. 3. Overall Inspection approach.

As it reaches to the target destination it will instruct the wrench motor to allow the SMR to reach to the location and obtain the current MFL reading of that place. These reading will be sent to the System. If an anomaly is detected then it is verified that the structure is having deformation at that particular location. If in vision based approach multiple anomalies have been detected then the robot will perform same procedure to determine the faults.

\section{Simulation results}

\subsection{Results for UAV}

The VBI from UAV has been performed by taking multiple images before and after the induced anomaly. The first set of images which was taken without anomaly are termed as images without crack (IoC), whereas the images taken after anomaly are termed as images with crack (IwC). These images are first stitched together and then the final stitched result of IoC and IwC are subtracted from each other to observe the presence of anomaly.

In this research the anomaly has been created by removing a screw from the structure. The stitched result for IoC and IwC along with the difference between them are shown in Fig. 4. It should be noted that the displaced screw has been clearly identified. 


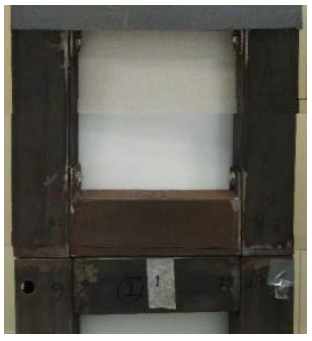

(a)

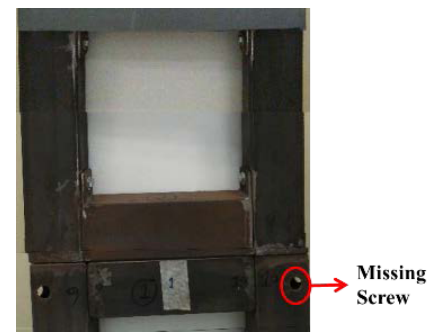

(b)

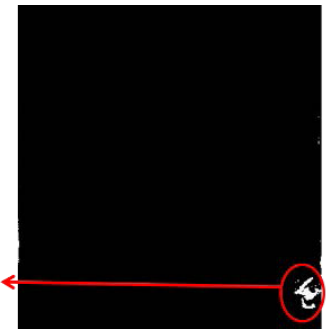

(c)

Fig. 4. Stitching results using stationary UAV for (a) IoC (b) IwC, and the crack identification (c) after filtering the subtraction result of (a) and (b).

\subsection{Results for MFL testing}

After detecting the presence of anomaly the respective GPS location along with the expected height has been sent to the FMR which directed the wrench motor and the SMR to move to that location and verify the presence of anomaly using MFL. The obtained result has been shown in Fig. 5, it should be noted that the graph has two peaks one is at the negative direction while the other is at the positive direction. Also, the negative peak comes before the positive one.

The reason of having a negative peak is because of the junction between two structural blocks as indicated clearly in Fig. 5(a). The structure which is used in this research consist of three cubes placed on each other. The place where the cubes boundaries touching each other have an inherent cavity at that point. At every junction the metal thickness will increased which decreases the magnetic field. Whereas, the positive peak is actually representing the absence of screw because the leakage in magnetic flux increase if there is an opening.

Although the negative field is false indication but this false indication will be marked for future so that the field change in these points will be ignored in future inspection. However if VBI indicates problem at the points of false indication then an inspector will be required to investigate that area.

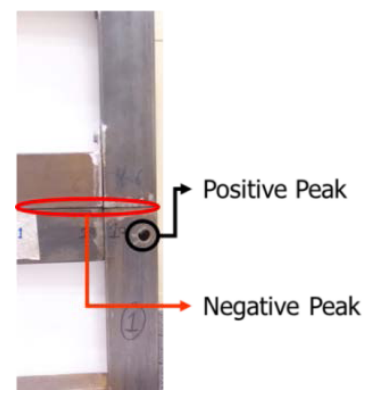

(a)

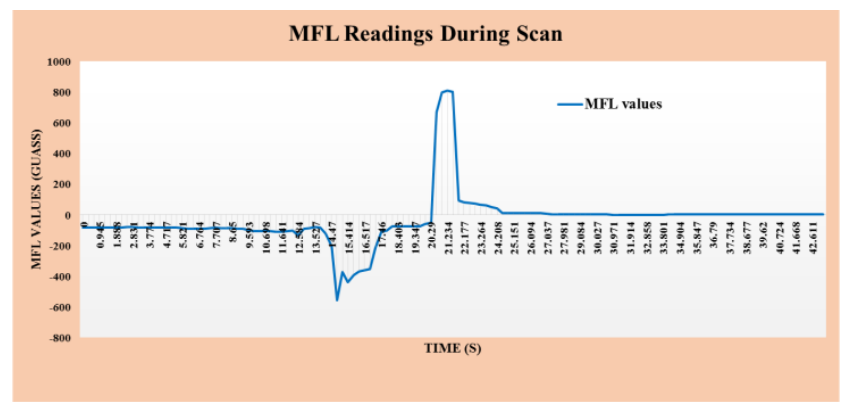

(b)

Fig. 5. Inspection results from MFL sensor.

\section{Conclusion}

The aim of this research is to develop coordinated robotic system for civil SHM. In order to achieve this objective, a UAV based VBI approach followed by a MFL based verification and localization has been adopted on a lab-grade sample structure. 
In the first round of scan, the UAVs ascertain the coarse defect signature using panoramic imaging. The structure has been scanned before and after the induced defect and the stitched image has been obtained for both scans. These two images after alignment has been compared and the defect which was in this research a missing screw has been identified. The GPS coordinates along with the height information of the defect has been sent to the FMR which after positioning itself with respect to the sent GPS values, will direct the SMR to reach to the location and perform the MFL based scanning of that region. The results obtained using MFL verified the defect which was located by the VBI.

With the initial findings, the proposed system appears to be a robust and inexpensive alternative to current approaches for automated inspection of civil/mechanical systems. The combination of VI and MFL approach provided the opportunity to detect, verify and localize the deformation in the structure.

This paper was made possible by National Priorities Research Program (NPRP) grant No. 7-234-2109 from the Qatar National Research Fund (a member of Qatar Foundation). The statements made herein are solely the responsibility of the authors.

\section{References}

1. The Human cost of weather related disasters, http://www.unisdr.org/files/46796_cop21weatherdisastersreport2015.pdf

2. F. N. Catbas, D. L. Brown, \& A. E. Aktan, "Parameter estimation for multiple-input multiple-output modal analysis of large structures", Journal of engineering mechanics, 130, 8, pp. 921-930 (2004)

3. S. W. Doebling, C. R. Farrar, \& M. B. Prime, "A summary review of vibration-based damage identification methods", Shock and vibration digest, 30, 2, pp. 91-105 (1998)

4. S. S. Ivanovic, M. D. Trifunac, \& M. I. Todorovska, "Ambient vibration tests of structures-a review”, ISET Journal of Earthquake Technology, 37, 4, pp. 165-197 (2000)

5. J. M. Caicedo, E. Clayton, S. J. Dyke, M. Abe, \& J. Tokyo, "Structural health monitoring for large structures using ambient vibrations", Proceedings of ICANCEER Conference, Hong Kong, pp. 379-384 (2002)

6. D. F. Mazurek, \& J. T. DeWolf, "Experimental study of bridge monitoring technique", Journal of Structural Engineering, 116, 9, pp. 2532-2549 (1990)

7. B. Glisic, D. Inaudi, J. M. Lau, \& C. C. Fong, "Ten-year monitoring of high-rise building columns using long-gauge fiber optic sensors", Smart Materials and Structures, 22, 5, pp. 055030 (2013)

8. H. R. Kess, \& D. E. Adams, "Investigation of operational and environmental variability effects on damage detection algorithms in a woven composite plate", Mechanical systems and signal processing, 21, 6, pp. 2394-2405 (2007)

9. M. R. Jahanshahi, \& S. F. Masri, "A new methodology for non-contact accurate crack width measurement through photogrammetry for automated structural safety evaluation", Smart materials and structures, 22, 3, pp. 035019 (2013)

10. Chen, ZhiQiang, and Jianfei Chen. "Mobile Imaging and Computing for Intelligent Structural Damage Inspection." Advances in Civil Engineering, 2014 (2014)

11. Z. Zhu, S. German, and I. Brilakis. "Detection of large-scale concrete columns for automated bridge inspection." Automation in construction, 19, 8, pp. 1047-1055 (2010)

12. A. Ellenberg, L. Branco, A. Krick, I. Bartoli, \& A. Kontsos. "Use of Unmanned Aerial Vehicle for Quantitative Infrastructure Evaluation". Journal of Infrastructure Systems, 21, 3, (2014)

13. Chaiyasarn, K., Kim, T. K., Viola, F., Cipolla, R., \& Soga, K. "Distortion-Free Image 
Mosaicing for Tunnel Inspection Based on Robust Cylindrical Surface Estimation through Structure from Motion". Journal of Computing in Civil Engineering, 04015045 (2015)

14. Bay, H., Tuytelaars, T., \& Van Gool, L., "Surf: Speeded up robust features", In Computer vision-ECCV 2006, Springer Berlin Heidelberg, pp. $404-417$ (2006)

15. Park, Seunghee, et al. "Magnetic flux leakage sensing-based steel cable NDE technique." Shock and Vibration, 2014 (2014)

16. Mandal, K., D. Dufour, and D. L. Atherton. "Use of magnetic Barkhausen noise and magnetic flux leakage signals for analysis of defects in pipeline steel." IEEE transactions on magnetics, 35, 3, pp. 2007-2017 (1999)

17. Phantom Pro-3: http://www.dji.com/product/phantom-3-pro. Accessed 4 Feb 2016

18. Vex Robotic Kit: http://www.vexrobotics.com/ 